\title{
Cycling Endurance of NOR Flash EEPROM Cells Under CHISEL Programming Operation-Impact of Technological Parameters and Scaling
}

\author{
Deleep R. Nair, Student Member, IEEE, S. Shukuri, and S. Mahapatra, Member, IEEE
}

\begin{abstract}
The impact of technological parameter (channel doping, source/drain junction depth) variation and channel length scaling on the reliability of NOR Flash EEPROM cells under channel initiated secondary electron (CHISEL) programming is studied. The best technology for CHISEL operation has been identified by using a number of performance metrics (cycling endurance of program/erase time, program/disturb margin) and scaling studies were done on this technology. It is explicitly shown that from a reliability perspective, bitcell optimization for CHISEL operation is quite different from that for channel hot electron (CHE) operation. Properly optimized bitcells show reliable CHISEL programming for floating gate length down to $0.2 \mu \mathrm{m}$.
\end{abstract}

Index Terms-Band-to-band tunneling, channel hot electron (CHE), channel initiated secondary electron (CHISEL), device scaling, drain disturb, Flash EEPROMs, hot carriers.

\section{INTRODUCTION}

C HANNEL INITIATED secondary electron (CHISEL) injection is an excellent programming mechanism for NOR Flash EEPROMs [1]-[5]. It relies on impact ionization feedback, is activated by the application of a negative substrate bias $\left(V_{B}\right)$ and provides high-energy electrons that get injected into the floating-gate (FG) over a spatially broad area in the channel [1]-[9], as schematically shown in Fig. 1. Compared to channel hot electron (CHE) injection [10], CHISEL injection provides lower voltage and lower power operation for equivalent programming time $\left(T_{P}\right)$ and faster $T_{P}$ under equivalent programming power. CHISEL injection also offers self-convergent programming leading to excellent threshold voltage $\left(V_{T}\right)$ control and a unique recovery procedure for over-erased cells [1]-[5], [9], [11], [12] not available under conventional CHE programming [13].

From the reliability perspective, CHISEL programming has shown good programmed and erased $V_{T}$ endurance up to $10^{5}$ program/erase (P/E) cycles [14], [15]. P/E cycling and data retention (after cycling) results obtained from large 32-Mbit arrays showed tight $V_{T}$ control and over 10 years of charge retention [14]. It has been clearly demonstrated that CHISEL

Manuscript received March 8, 2004; revised July 14, 2004. The review of this paper was arranged by Editor R. Shrivastava.

D. R. Nair and S. Mahapatra are with the Department of Electrical Engineering, Indian Institute of Technology Bombay, Mumbai 400076, India (e-mail: drnair@ee.iitb.ac.in).

S. Shukuri was with Renesas Technology Corporation, Tokyo, Japan. He is now with Genusion Inc., Amagasaki, Japan.

Digital Object Identifier 10.1109/TED.2004.835996

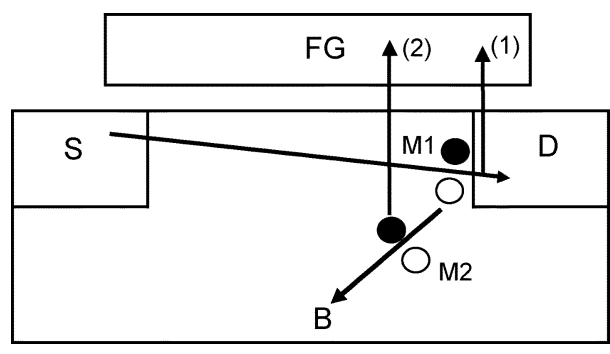

Fig. 1. CHISEL injection mechanism (schematic). Channel electrons, heated by lateral electric field create primary electron-hole pairs by impact ionization (M1). Primary holes flow to the substrate and in the presence of high transverse electric field (due to negative substrate bias) create secondary electron-hole pairs by impact ionization (M2). The secondary electrons move toward the interface and those having energy greater than $3.1 \mathrm{eV}$ get injected into the FG.

programming is free from anomalous bit failure through increased disturbs, window closure, or charge loss.

From the cell scaling perspective, a few earlier reports indicated degradation in CHISEL programming efficiency for deeply scaled cells [16], [17]. However, it has been recently demonstrated, albeit on pre-cycled cells, that it is possible to maintain excellent CHISEL programming efficiency for cells having FG length $\left(L_{\mathrm{FG}}\right)$ down to $0.2 \mu \mathrm{m}$ with proper adjustment of technological parameters [18]. However, to the best of our knowledge, the impact of technological parameters and cell scaling on cycling endurance has not been reported so far for CHISEL programming operation.

This scope of paper is two fold. First, the best cell design for reliable CHISEL operation is identified from a number of technology parameter options (variation in channel doping and junction depth and option of halo doping). Cycling induced degradation in $T_{P}$, erase time $\left(T_{E}\right), \mathrm{P} / \mathrm{E} V_{T}\left(V_{\mathrm{TP}}\right.$ and $\left.V_{\mathrm{TE}}\right)$ and program/disturb (P/D) margin were used as metrics for cell selection. It is shown that halo doping is not a good option since those cells suffer from severe degradation of $T_{E}$ and P/D margin after cycling. On the other hand, nonhalo cells with shallow junction depth suffer from degradation in P/D margin. The best tradeoff between $T_{P}$ and P/D margin was obtained for nonhalo cells with higher channel doping but somewhat deeper junction. It is also explicitly shown that the best cell design for reliable CHISEL operation is quite different from that under CHE operation. Second, the impact of $L_{\mathrm{FG}}$ scaling on the cycling endurance of the above reliability metrics was studied for the optimized cell. For the present choice of technological parameters, reliable CHISEL programming operation is achieved for cells having $L_{\mathrm{FG}}$ down to $0.2 \mu \mathrm{m}$. 
TABLE I

DEVICES USED IN THIS STUdy. $V_{T}\left(V_{\mathrm{CG}}\right.$ FOR $\left.I_{D} / V_{D}=5 \mu \mathrm{A} / 0.8 \mathrm{~V}\right)$ AND $V_{\mathrm{BD}}\left(V_{D}\right.$ FOR $\left.I_{D}=1 \mu \mathrm{A}, V_{\mathrm{CG}}=0\right)$ MEASURED ON IDENTICAL FG-CG SHORTED FETS

\begin{tabular}{l|l|l|l|l|l}
\hline Device & $\begin{array}{l}\text { Channel } \\
\text { Implant }\end{array}$ & $\begin{array}{l}\text { Junction } \\
\text { Depth }\end{array}$ & Halo & $\begin{array}{l}\text { Natural } \\
\mathrm{V}_{\mathrm{T}}(\mathrm{V})\end{array}$ & $\begin{array}{l}\text { Junction } \\
\mathrm{V}_{\mathrm{BD}}(\mathrm{V})\end{array}$ \\
\hline LL & Low & Low & No & 2.11 & 7.2 \\
\hline HH & High & High & No & 2.21 & 7.3 \\
\hline HL & High & Low & No & 2.37 & 7.0 \\
\hline Halo & Very low & Very high & Yes & 2.43 & 6.6 \\
\hline
\end{tabular}

\section{EXPERIMENTAL}

The devices used in this work were fabricated using a state-ofthe-art $0.18-\mu \mathrm{m}$ triple well process featuring advanced modules such as shallow trench isolation and self-aligned source/drain (S/D) contacts leading to cell area of about $0.45 \mu \mathrm{m}^{2}$. Measurements were performed on isolated, fully scaled bitcells having finished $L_{\mathrm{FG}}$ of 0.32 through $0.2 \mu \mathrm{m}$, width $(W)$ of $0.3-\mu \mathrm{m}$, tunnel oxide $\left(T_{\mathrm{OX}}\right)$, and oxide-nitride-oxide interpoly dielectric thickness of 12 and $20 \mathrm{~nm}$, respectively, and gate coupling of about 0.55 . Four different types of cells (LL, HH, HL, and Halo) were fabricated to study the impact of changes in technological parameters on CHISEL program and disturb performance, before and after P/E cycling. Their doping schemes, junction depth, natural $V_{T}$, and junction breakdown voltage $\left(V_{\mathrm{BD}}\right)$ are mentioned in Table I.

This paper focuses on CHISEL programming, which was performed using $V_{B}=-2 \mathrm{~V}$ (source grounded). For comparison, CHE programming was performed for select cases using $V_{B}=0 \mathrm{~V}$. The cells were erased using uniform channel erase with source, drain and substrate grounded. $V_{T}$ was defined as the control gate bias $\left(V_{\mathrm{CG}}\right)$ required to obtain $5-\mu \mathrm{A}$ drain current $\left(I_{D}\right)$ at $0.8-\mathrm{V}$ drain bias $\left(V_{D}\right)$. Programmed and erased levels are defined as $V_{\mathrm{TP}}=5.4 \mathrm{~V}$ and $V_{\mathrm{TE}}=1.9 \mathrm{~V}$, respectively. Drain disturb [19] measurements were performed at identical $V_{D}$ and $V_{B}$ as programming, but with zero $V_{\mathrm{CG}}$ to simulate an unselected $W L$. Drain disturb is studied for both the charge gain (erased cell) and charge loss (programmed cell) modes. Disturb time $T_{D}$ is defined as time for a $V_{T}$ change of $0.1 \mathrm{~V}$ during disturb transients. P/E cycling was done under fixed $T_{P}$ and $T_{E}$ as the initial cell.

\section{RESULTS AND DISCUSSION}

\section{A. Comparison Between Halo and Nonhalo Cells}

Fig. 2 shows the schematic representation of the halo and nonhalo cells used in the study. Nonhalo cells have lower channel doping, a shallower S/D junction and no halo implant. Halo cells have lower channel doping, a deeper S/D junction anda heavy halo implant. Fig. 3 shows CHISEL program and erase transients for halo and nonhalo (LL) $L_{\mathrm{FG}}=0.26 \mu \mathrm{m}$ cells before and after $100 \mathrm{~K} \mathrm{P} / \mathrm{E}$ cycles under identical bias. Initial $T_{P}$ and $T_{E}$ are slightly lower for the halo cell. It can be clearly seen that the degradation of programming transients (and $T_{P}$ ) after cycling is comparable (and negligible) for both technologies. Note
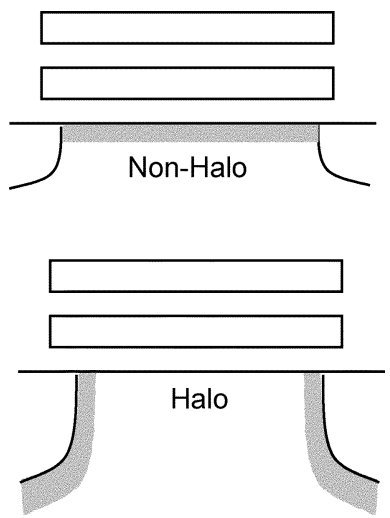

Fig. 2. Schematic representation of the two type of Flash cells used in the study. Nonhalo cells have moderate to high channel doping, shallower S/D junction and no halo implant. Halo cells have lower channel doping, deeper S/D junction and heavy halo implant.

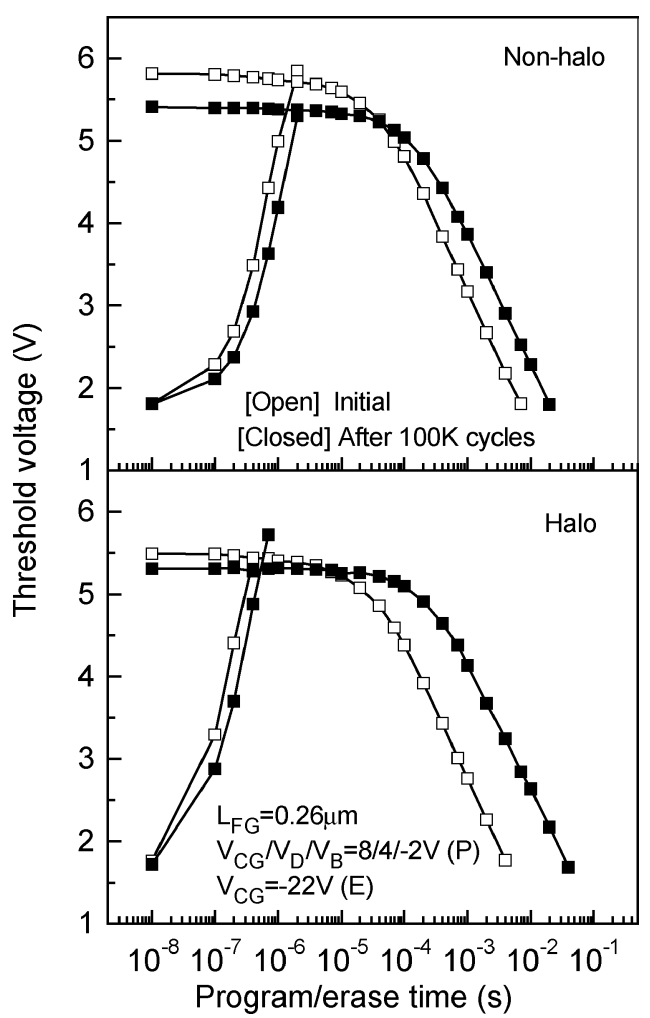

Fig. 3. P/E transients measured under equivalent bias on nonhalo (LL) and halo cells before and after $100 \mathrm{~K} \mathrm{P} / \mathrm{E}$ cycles.

that such negligible cycling induced degradation of programming transients is a standard feature of CHISEL programming operation [14], [15]. However, the halo device shows higher degradation in erase time compared to the nonhalo device.

Fig. 4 shows the P/E cycling induced degradation of programmed and erased $V_{T}$ for halo and nonhalo (LL) $L_{\mathrm{FG}}=$ $0.26 \mu \mathrm{m}$ cells under identical bias as in Fig. 3. Identical bias condition was chosen for comparison, as there exists different combination of $V_{\mathrm{CG}}$ and $V_{D}$ to get the same $T_{P}$ [18]. $V_{\mathrm{TP}}$ degradation is always insignificant for both the cells while the halo cell shows higher $V_{\mathrm{TE}}$ degradation, consistent with $T_{P}$ and $T_{E}$ degradation shown in Fig. 3. It is well known that the electron 


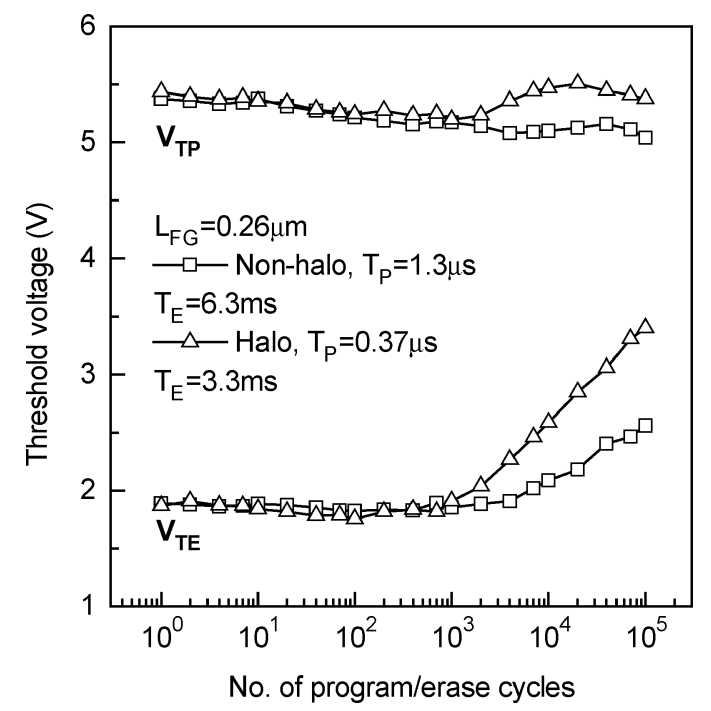

Fig. 4. Cycling endurance of program and erase $V_{T}$ measured under equivalent bias (as in Fig. 3) on nonhalo (LL) and halo cells.

energy distribution (EED) high-energy tail under CHISEL operation is always populated due to secondary impact ionization [1]-[9]. Hence, electrons that constitute the gate current $\left(I_{G}\right)$ during programming can easily overcome the increased injection barrier caused by cycling induced negative bulk and interfacial charges and results in negligible $T_{P}$ degradation [14], [15]. It is also well known that CHISEL injection takes place over a broader area (toward the channel) and the resultant broader area of defect generation affects electron-ejection during erase and degrades $T_{E}$ (compared to CHE operation) [15]. Higher $T_{E}$ degradation for halo cells indicates higher (or wider) defect generation in the channel for such cells during cycling under CHISEL programming operation.

Figs. 5 and 6, respectively, show program and erase time before and after $100 \mathrm{~K} \mathrm{P} / \mathrm{E}$ cycles as a function of FG length for halo and nonhalo (LL) cells. Program and erase were done at fixed biases for all $L_{\mathrm{FG}}$ values. The halo cells shows much better $T_{P}$ scaling and comparable $T_{P}$ degradation after cycling compared to the nonhalo cells. The faster programming clearly indicates higher hot electron generation for halo cells under identical bias (expected because of higher doping around the junction). The halo cells also show better initial $T_{E}$. However, they suffer from severe $T_{E}$ degradation after cycling (specially at smaller $L_{\mathrm{FG}}$ ) compared to the nonhalo cells. This is expected since a wider defect generation area is likely to degrade a significant part of the channel for smaller $L_{\mathrm{FG}}$ halo cells and therefore cause higher degradation in $T_{E}$ after cycling.

Fig. 7 shows the drain disturb time before and after $100 \mathrm{~K}$ $\mathrm{P} / \mathrm{E}$ cycles as a function of FG length for halo and nonhalo (LL) cells, under both charge gain and loss disturb modes. It has been previously identified that unlike CHE operation, CHISEL operation shows both charge gain and loss disturb that originates from band-to-band tunneling (BTBT) [19]. BTBT generated electrons (holes) get heated by the junction field and get attracted toward the FG by positive FG charge in erased state (by negative FG charge during erase state) and cause charge gain (charge loss) disturb. Note that increased disturb implies lower $T_{D}$ and vice-versa. It can be clearly seen that charge gain

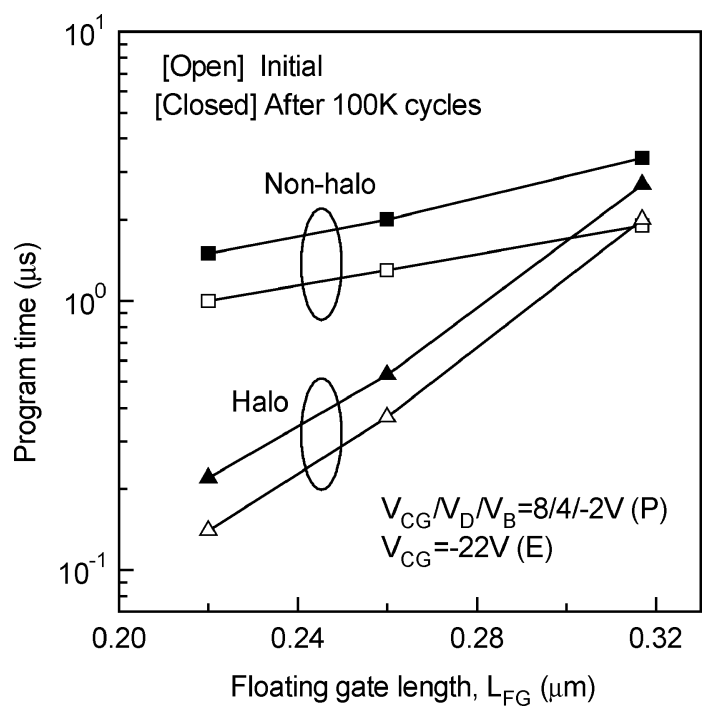

Fig. 5. Program time $\left(T_{P}\right)$ as a function of FG length $\left(L_{\mathrm{FG}}\right)$ measured under equivalent bias on nonhalo (LL) and halo cells before and after $100 \mathrm{~K} \mathrm{P} / \mathrm{E}$ cycles.

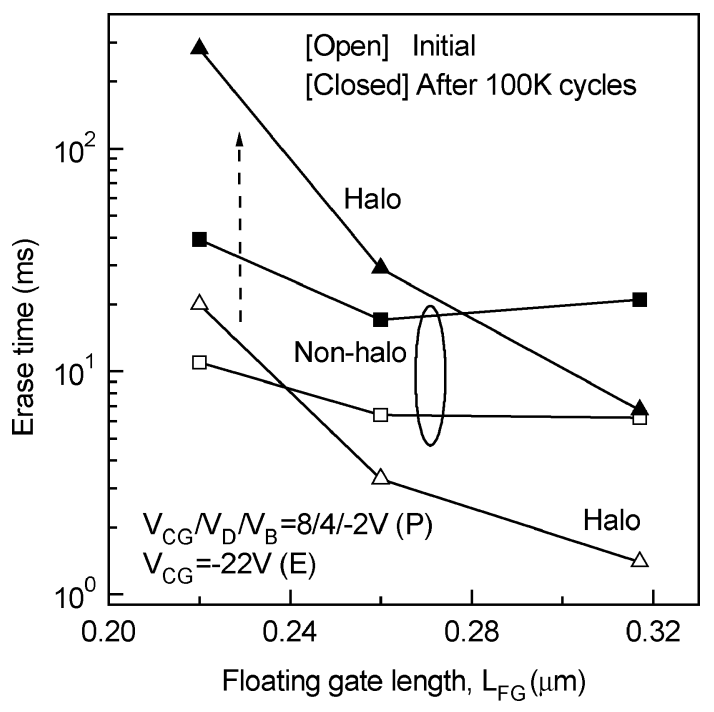

Fig. 6. Erase time $\left(T_{E}\right)$ as a function of FG length $\left(L_{\mathrm{FG}}\right)$ measured under equivalent bias on nonhalo (LL) and halo cells before and after $100 \mathrm{~K} \mathrm{P} / \mathrm{E}$ cycles.

disturb increases but charge loss disturb decreases after P/E cycling. Such opposite cycling induced degradation of charge gain and loss disturb under CHISEL operation has already been observed and explained [20], [21]. The cycling induced degradation in $T_{D}$ is higher for halo cells compared to the nonhalo cells, which is consistent with higher defect generation for the former device. The drastic increase in charge gain disturb after cycling specially at smaller $L_{\mathrm{FG}}$ for halo cells (resulting in a P/D margin of $\sim 10^{4}$ at $L_{\mathrm{FG}}=0.21 \mu \mathrm{m}$ ) is a very severe concern.

The above results show that halo cells, despite showing quite promising $T_{P}$ scaling, fare badly after cycling due to severe degradation in $T_{E}$ and P/D margin. This forces us to abort the halo cells and find out the best among the nonhalo technologies, which is done in the next section.

\section{B. Optimization of Nonhalo Cells}

All the nonhalo technologies (LL, HH and HL, L = low, $\mathrm{H}$ $=$ high, the letters correspond to channel doping and junction 


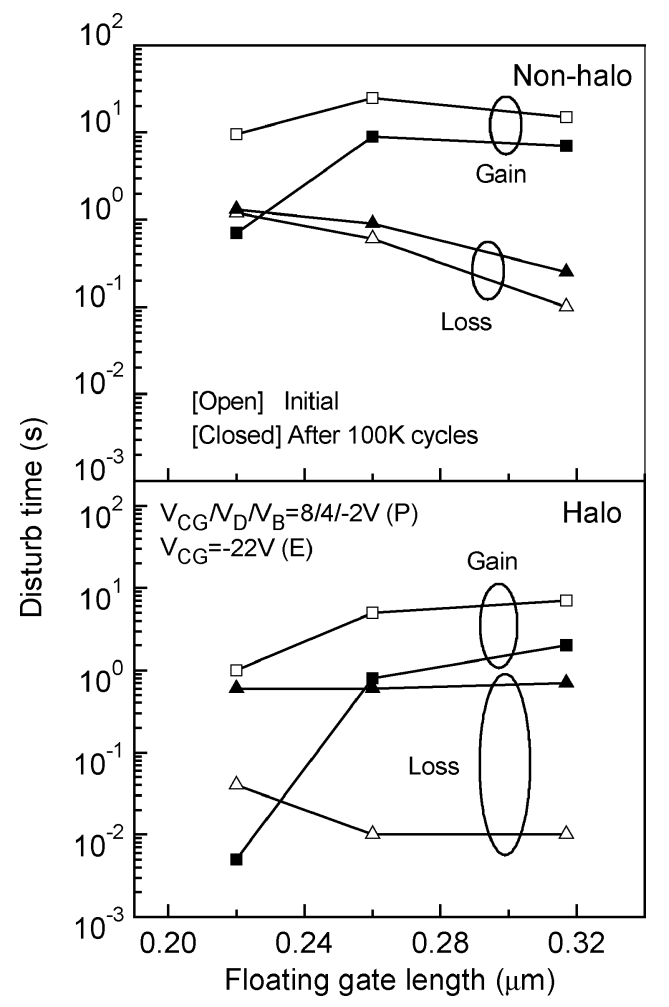

Fig. 7. Disturb time $\left(T_{D}\right)$ as a function of FG length $\left(L_{\mathrm{FG}}\right)$ measured under equivalent bias on nonhalo (LL) and halo cells before and after $100 \mathrm{~K} \mathrm{P/E} \mathrm{cycles.}$

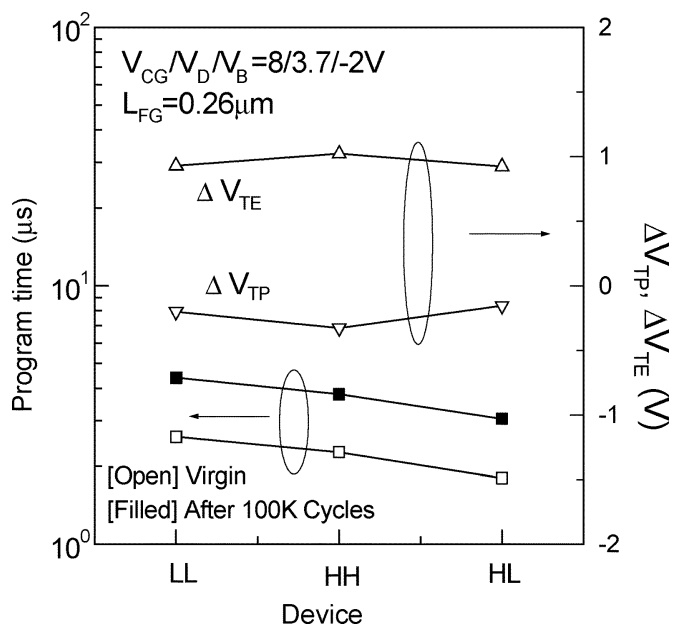

Fig. 8. Impact of technology parameters on cycling endurance of program time and programmed and erased $V_{T}$ for nonhalo cells under CHISEL operation.

depth, respectively) were compared under identical program and erase biases.

Fig. 8 shows the programming time before and after $100 \mathrm{~K}$ $\mathrm{P} / \mathrm{E}$ cycles and corresponding degradation in programmed and erased $V_{T}\left(\Delta V_{\mathrm{TP}}\right.$ and $\left.\Delta V_{\mathrm{TE}}\right)$ for different nonhalo technologies under CHISEL operation. Note that initial $T_{P}$ reduces from LL to HL cells because of increasing transverse field under identical programming bias [18]. However, the cycling induced degradation in $T_{P}, V_{\mathrm{TP}}$ and $V_{\mathrm{TE}}$ (and $T_{E}$, not shown) are insensitive to changes in technological parameters. Similar $T_{P}$ and $V_{\mathrm{TP}}$ degradation does not necessarily suggest identical cycling induced degradation across different technologies. This is

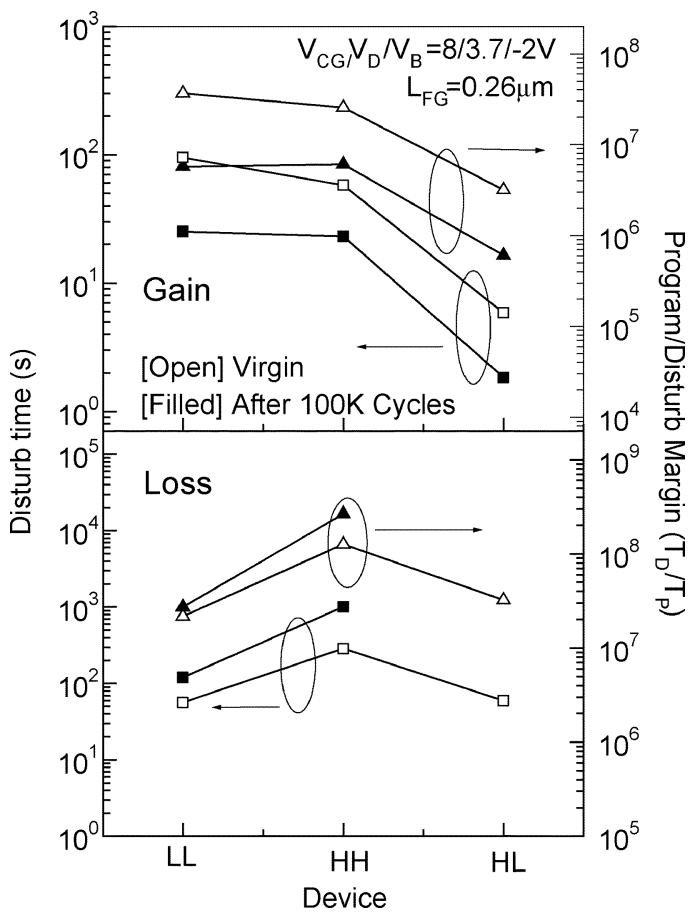

Fig. 9. Impact of technology parameters on the cycling endurance of disturb time and program/disturb margin for nonhalo cells under CHISEL operation.

because the high-energy secondary electrons can easily overcome any moderate differences in injection barrier (caused by moderate differences in cycling induced degradation) across different technologies. However, similar $V_{\mathrm{TE}}$ degradation (note that erase is by Fowler-Nordheim electron ejection) suggests similar cycling induced degradation during CHISEL operation across various technologies.

Fig. 9 shows the disturb time and P/D margin before and after $100 \mathrm{~K} \mathrm{P} / \mathrm{E}$ cycles for different nonhalo technologies under CHISEL operation. The device HL did not show detectable charge loss after P/E cycling within the measurement timescale. Charge loss disturb is always less compared to the charge gain disturb for all technologies. Furthermore, charge gain disturb increases while charge loss disturb decreases after $\mathrm{P} / \mathrm{E}$ cycling. The P/D margin is therefore an important concern only for the charge gain mode. It can be seen from the figure that both LL and HH cells show comparable P/D margin while that for HL cells are lower both before and after $100 \mathrm{~K}$ cycles. Since CHISEL drain disturb originates from BTBT, HL cells with highest transverse field results in lowest $T_{D}$ (which also showed fastest $T_{P}$ during programming). However, $T_{D}$ degradation is similar after P/E cycling for all technologies. This is expected since cycling induced degradation has been found to be similar across different technologies.

By comparing the degradation in $T_{P}$ and $\mathrm{P} / \mathrm{D}$ margin after cycling under CHISEL operation, the best technology is identified to be $\mathrm{HH}$. This technology is chosen for studying CHISEL $L_{\mathrm{FG}}$ scaling in the next section.

Note that CHISEL mechanism is substantially different from the conventional CHE process. It is therefore expected that the best-optimized technology for CHISEL operation need not be the same for CHE operation and vice-versa. Fig. 10 shows the 


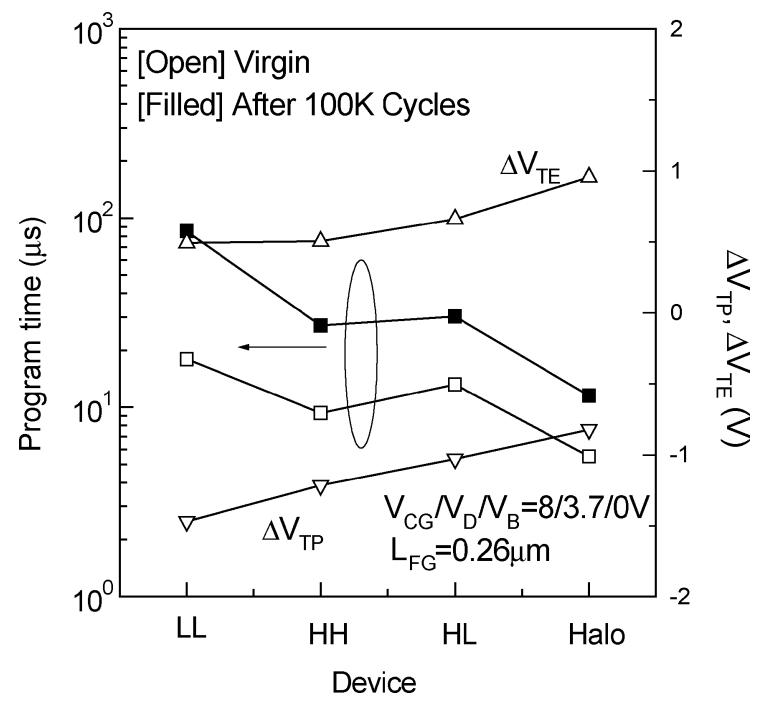

Fig. 10. Impact of technology parameters on cycling endurance of program time and programmed and erased $V_{T}$ for nonhalo cells under CHE operation $\left(V_{B}=0 \mathrm{~V}\right)$.

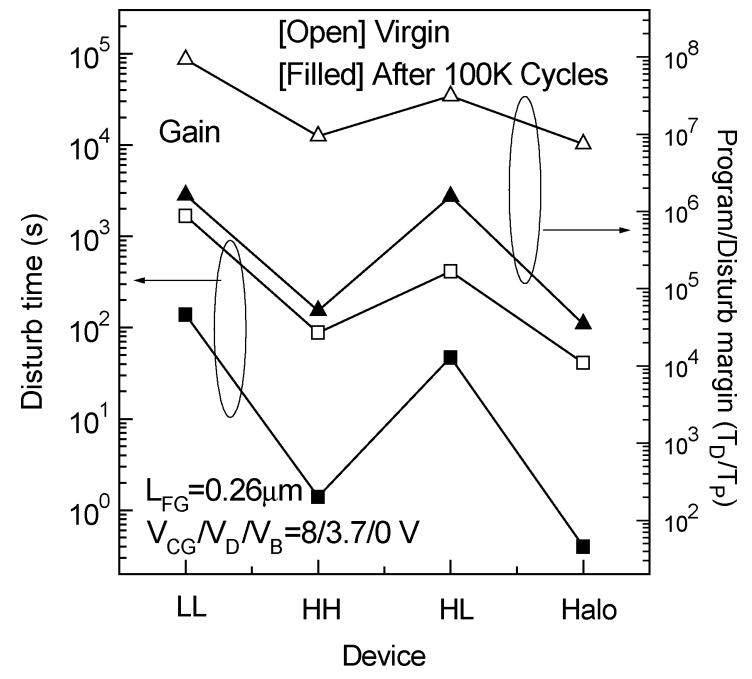

Fig. 11. Impact of technology parameters on the cycling endurance of disturb time and program/disturb margin for nonhalo cells under CHE operation.

programming time before and after $100 \mathrm{~K} \mathrm{P} / \mathrm{E}$ cycles and corresponding degradation in programmed and erased $V_{T}$ for all nonhalo and halo technologies under $\mathrm{CHE}$ operation. The programming was done at identical $V_{\mathrm{CG}}$ and $V_{D}$. It is important to note that unlike CHISEL operation there is a wide variation in degradation across different technologies under CHE operation. Halo cells show fastest initial $T_{P}$ and lowest $T_{P}$ and $V_{\mathrm{TP}}$ degradation but highest $V_{\mathrm{TE}}$ degradation after cycling. On the other extreme, nonhalo LL cells show worst initial $T_{P}$ and highest $T_{P}$ and $V_{\mathrm{TP}}$ degradation but lowest $V_{\mathrm{TE}}$ degradation after cycling. The degradation of HH and HL cells fall between these two extremes.

Fig. 11 shows the impact of technology parameters on charge gain drain disturb and P/D margin before and after $100 \mathrm{~K} \mathrm{P} / \mathrm{E}$ cycles under CHE operation. Note that CHE shows charge loss disturb at much higher $V_{D}$ values than used in this work [19]. Contrary to CHISEL operation, cells with lower junction depth show lower initial and degraded $T_{D}$ and correspondingly higher $\mathrm{P} / \mathrm{D}$ margin. Unlike CHISEL operation, the HL technology fares

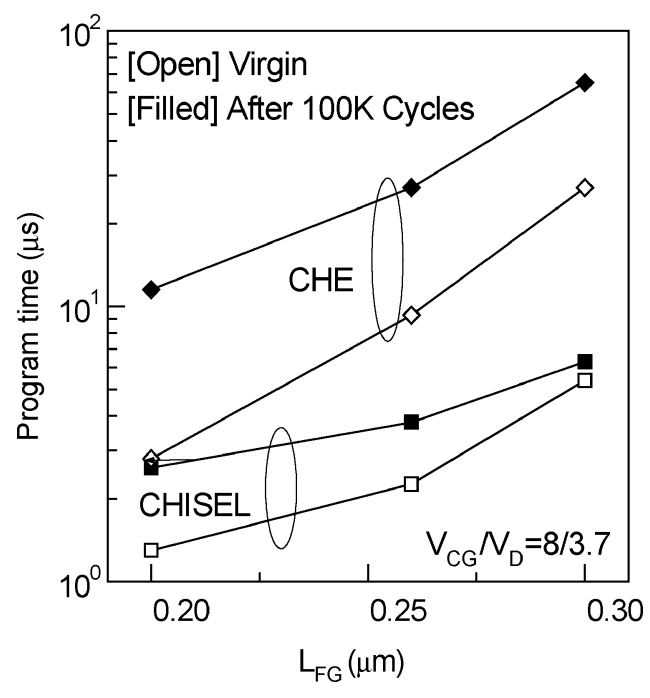

Fig. 12. Impact of $L_{\mathrm{FG}}$ scaling on the cycling endurance of program time for nonhalo $\mathrm{HH}$ cells under CHISEL and $\mathrm{CHE}$ operation.

best (among the available choices explored in the present work) in terms of $T_{P}$ and P/D margin degradation under CHE operation. This clearly shows that the Flash memory cell optimization should be treated differently for CHE and CHISEL operation.

\section{Effect of Scaling}

In the previous section, it has been identified that $\mathrm{HH}$ nonhalo technology shows the most reliable CHISEL operation. In this section the scaling performance of this technology is explored. The program and erase biases were held fixed as $L_{\mathrm{FG}}$ is varied, which yields faster $T_{P}$ at smaller $L_{\mathrm{FG}}$. Comparison under identical $T_{P}$ (by suitably adjusting $V_{\mathrm{CG}}$ and $V_{D}$ ) is beyond the scope of the present paper. Furthermore, studying the scalability of the best optimized CHE cell is also beyond the scope of the present paper.

Fig. 12 shows the programming time before and after P/E cycles as a function of FG length under CHISEL operation. For comparison, the cycling induced $T_{P}$ degradation under $\mathrm{CHE}$ operation is also shown. Note that $T_{P}$ degradation after $100 \mathrm{~K}$ cycles increases as $L_{\mathrm{FG}}$ is reduced. This is expected since at constant $V_{D}$ smaller $L_{\mathrm{FG}}$ cells are expected to degrade by a large amount, despite lower $T_{P}$ during cycling. However, the degradation under CHISEL operation is much smaller than that under CHE operation. It has been shown before [22] that CHISEL operation results in a highly populated EED high-energy tail even at shorter $L_{\mathrm{FG}}$. These highly energetic CHISEL electrons can easily overcome the increased energy barrier caused by trapping of electrons during cycling. This is reflected in lower degradation of $T_{P}$ as shown.

Fig. 13 shows charge gain and loss drain disturb and corresponding P/D margin as a function of FG length before/after P/E cycling under CHISEL operation. Charge loss disturb decreases at smaller $L_{\mathrm{FG}}$ (increase in drain coupling makes FG voltage less negative and reduces BTBT). As mentioned before, charge loss disturb also decreases after cycling. The corresponding P/D margin is improved and therefore is not an issue during CHISEL cell scaling. Charge gain disturb is less sensitive to $L_{\mathrm{FG}}$ before cycling under CHISEL operation [19]. However, it increases and hence $\mathrm{P} / \mathrm{D}$ margin reduces after cycling as $L_{\mathrm{FG}}$ is scaled. 


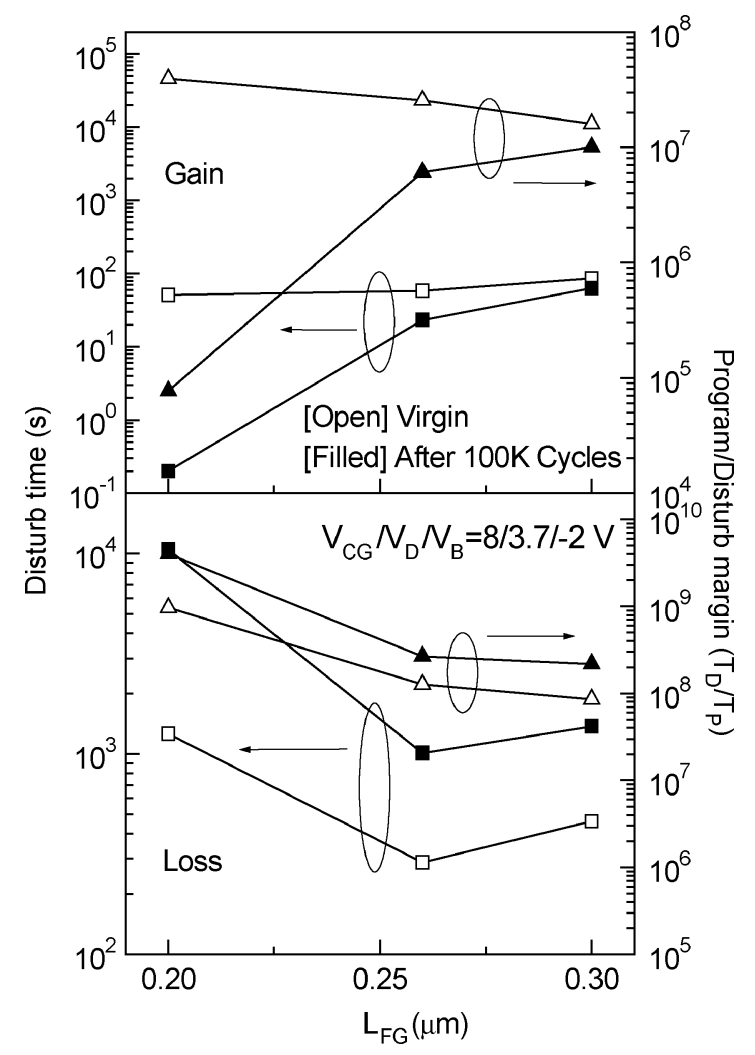

Fig. 13. Impact of $L_{\mathrm{FG}}$ scaling on the cycling endurance of disturb time and program/disturb margin for nonhalo $\mathrm{HH}$ cells under CHISEL operation.

This is due to higher cycling induced degradation (since $V_{D}$ is kept constant) at lower $L_{\mathrm{FG}}$. Therefore charge gain disturb poses some concern for $L_{\mathrm{FG}}$ scaling during CHISEL operation.

For the present $\mathrm{HH}$ technology optimized for $L_{\mathrm{FG}}=$ $0.26 \mu \mathrm{m}$, a $L_{\mathrm{FG}}=0.2 \mu \mathrm{m}$ cell shows $T_{P}=2.6 \mu \mathrm{s}$ and P/D margin greater than $5 \times 10^{4}$ after $100 \mathrm{~K} \mathrm{P/E}$ cycles under CHISEL operation at $V_{D}=3.7 \mathrm{~V}$.

\section{CONCLUSION}

To summarize, we have studied the impact of variation in technological parameters (channel doping, S/D junction depth) and channel length scaling on the reliability of NOR Flash EEPROM cells under CHISEL programming operation. Cycling induced degradation of $\mathrm{P} / \mathrm{E}$ time and program/disturb margin were used as performance metric. It has been shown that halo doped cells, despite showing excellent initial performance are not reliable after cycling. Nonhalo cells with high channel doping and somewhat high S/D junction depth has been found to be most suitable for reliable CHISEL operation. It has been also shown that bitcell optimization for reliable CHISEL operation is quite different from that for CHE operation. Under present condition, the best-optimized nonhalo cells show reliable CHISEL programming for FG length down to $0.2 \mu \mathrm{m}$. These results prove that CHISEL programming can be reliably used in deeply scaled NOR Flash EEPROM cells.

\section{REFERENCES}

[1] J. D. Bude, A. Frommer, M. R. Pinto, and G. R. Weber, "EEPROM/flash sub-3.0 V drain-source bias hot carrier writing," in IEDM Tech. Dig., 1995, pp. 989-991.
[2] J. D. Bude et al., "Secondary electron Flash—a high performance low power Flash technology for $0.35 \mu \mathrm{m}$ and below," in IEDM Tech. Dig., 1997, pp. 279-282.

[3] M. Mastrapasqua, "Low voltage Flash memory by use of a substrate bias," Microelectron. Eng., vol. 48, pp. 389-394, 1999.

[4] D. Esseni, A. D. Strada, P. Cappelletti, and B. Ricco, "A new and flexible scheme for hot-electron programming of nonvolatile memory cells," IEEE Trans. Electron Devices, vol. 46, pp. 125-133, Jan. 1999.

[5] S. Mahapatra, S. Shukuri, and J. D. Bude, "CHISEL Flash EEPROM-Part 1: Performance and scaling," IEEE Trans. Electron Devices, vol. 49, pp. 1296-1301, July 2002.

[6] J. D. Bude, "Gate current by impact ionization feedback in sub-micron MOSFET technologies," in Proc. Symp. VLSI Tech., 1995, pp. 101-102.

[7] D. Esseni and L. Selmi, "A better understanding of substrate enhanced gate current in MOSFETs and Flash cells-Part I: Phenomenological aspects," IEEE Trans. Electron Devices, vol. 46, pp. 369-375, Feb. 1999.

[8] L. Selmi and D. Esseni, "A better understanding of substrate enhanced gate current in MOSFETs and Flash cells-Part II: Physical analysis," IEEE Trans. Electron Devices, vol. 46, pp. 376-382, Feb. 1999.

[9] J. D. Bude, M. R. Pinto, and R. K. Smith, "Monte Carlo simulation of CHISEL Flash memory cell," IEEE Trans. Electron Devices, vol. 47, pp. 1873-1881, Oct. 2000.

[10] P. Cappelletti, C. Golla, P. Olivo, and E. Zanoni, Flash Memories. Norwell, MA: Kluwer, 1999.

[11] C. Y. Hu et al., "A convergence scheme for over erased Flash EEPROMs using substrate enhanced hot electron injection," IEEE Electron Device Lett., vol. 11, pp. 500-502, 1995.

[12] — "Substrate-current-induced hot electron (SCIHE) injection: Anew convergence scheme for Flash memory," in IEDM Tech. Dig., 1995, pp. 283-286.

[13] K. Yoshikawa et al., "Comparison of current Flash EEPROM erasing methods: stability and how to control," in IEDM Tech. Dig., 1992, pp. 595-598.

[14] S. Mahapatra, S. Shukuri, and J. D. Bude, "CHISEL Flash EEPROM-Part 2: Reliability," IEEE Trans. Electron Devices, vol. 49, pp. 1302-1307, July 2002.

[15] N. R. Mohapatra, S. Mahapatra, V. R. Rao, J. Vasi, S. Shukuri, and J. D. Bude, "Effect of programming biases on the reliability of CHE and CHISEL Flash EEPROMs," in Proc. IRPS, 2003, pp. 518-522.

[16] D. Esseni, L. Selmi, A. Ghetti, and E. Sangiorgi, "The scaling properties of CHISEL and CHE injection efficiency in MOSFETs and Flash memory devices," in IEDM Tech. Dig., 1999, pp. 275-279.

[17] — - "Injection efficiency of CHISEL gate currents in short MOS devices: physical mechanisms, device implications, and sensitivity to technological parameters," IEEE Trans. Electron Devices, vol. 47, pp. 2194-2200, Nov. 2000.

[18] N. R. Mohapatra, D. R. Nair, S. Mahapatra, V. R. Rao, S. Shukuri, and J. D. Bude, "CHISEL programming operation of scaled NOR Flash EEPROMs - effect of voltage scaling, device scaling and technological parameters," IEEE Trans. Electron Devices, vol. 50, pp. 2104-2111, Oct. 2003.

[19] D. R. Nair, S. Mahapatra, S. Shukuri, and J. D. Bude, "Drain disturb during CHISEL programming of NOR Flash EEPROMs-physical mechanism and impact of scaling and technological parameters," IEEE Trans. Electron Devices, vol. 51, pp. 701-707, May 2004.

[20] D. R. Nair, N. R. Mohapatra, S. Mahapatra, S. Shukuri, and J. D. Bude, "The effect of CHE and CHISEL programming operation on drain disturb in Flash EEPROMs," in Proc. IPFA, 2003, pp. 164-168.

[21] — "Effect of P/E cycling on drain disturb in Flash EEPROM's under CHE and CHISEL operation," IEEE Trans. Devices Mater. Reliabil., vol. 4, pp. 32-37, March 2004.

[22] D. R. Nair, N. R. Mohapatra, S. Mahapatra, S. Shukuri, and J. Bude, "Effect of scaling on the reliability of Flash EEPROMs under CHISEL programming," in Proc. SISC , 2003, p. P17.

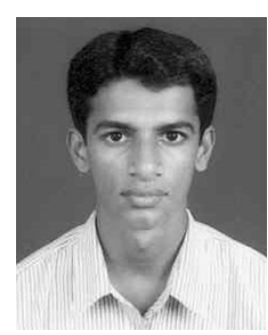

Deleep R. Nair (S '98) was born in New Delhi, India, in 1978. He received the B.Tech. degree in electronics and communication engineering from REC Calicut, India, in 1999, and the M.Tech. degree in electrical engineering from the Indian Institute of Technology (IIT), Bombay, India, in 2001. Since 2001, he has been pursuing the Ph.D. degree at IIT, Bombay.

His current interests include MOS physics and technology, characterization and numerical simulation of semiconductor devices. He has worked on the characterization and simulation of Flash memories and numerical modeling of quantum effects in MOS devices. 


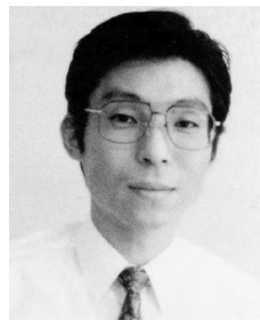

S. Shukuri was born in Oita, Japan, in 1958. $\mathrm{He}$ received the B.S. degree in electrical engineering from Yamanashi University, Yamanashi, Japan, in 1980 and the M.S. degree in electrical engineering from Kyushu University, Fukuoka, Japan, in 1982.

In 1982, he joined Central Research Laboratory, Hitachi Ltd., Tokyo, Japan, where he engaged in the research of the focused ion beam implantation and its application to LSIs. From 1987 to 1992, he has engaged in the process and device design of highspeed BiCMOS and DRAM cell. In 1993 he joined the Semiconductor and Integrated Circuits Division, Hitachi Ltd., where he has been in charge of Flash memory device development.

Mr. Shukuri is a member of the Japan Society of Applied Physics.

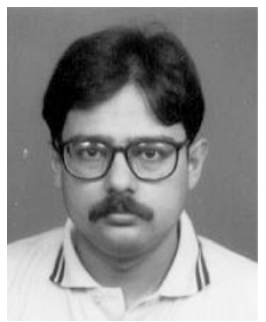

S. Mahapatra (M'99) received the M.Sc. degree in physics from Jadavpur University, Calcutta, India and the Ph.D. degree in electrical engineering (microelectronics) from the Indian Institute of Technology (IIT), Bombay, India, in 1995 and 1999 respectively. His doctoral thesis was on the study of hot-carrier degradation in conventional, channel engineered and high- $\kappa$ MOSFETs using a novel charge pumping technique.

From 2000 to 2001 he was at Bell Laboratories, Lucent Technologies, Murray Hill, NJ. At Bell Labs, he played a key role in designing and developing the unit cell of the world's first commercial CHISEL Flash memory, and was also involved in studies of interface characterization of GaAs-GdO FETs, hot-carrier instability in RFLDMOS and p-MOSFET bias temperature instability. Since January 2002, he has been with the IIT Department of Electrical Engineering, where he is presently an Assistant Professor. His present research interest involves semiconductor device physics, simulation, modeling and characterization, novel devices, hot-carrier and bias temperature reliability issues in MOSFETs, Flash memories and high- $\kappa$ gate dielectrics. He has published more than 30 papers in refereed international journals and conferences, and worked as a reviewer for many international journals and conferences. 